https://doi.org/10.48009/2_iis_2009_505-512

\title{
BLENDING DECISION AND DESIGN SCIENCE WITH INFORMATION SYSTEMS DESIGN AS A MEANS TO OPTIMIZING BUSINESS DECISIONS
}

\author{
Gina Boff, California University of Pennsylvania, boff@cup.edu \\ Lisa Kovalchick, California University of Pennsylvania, kovalchick@cup.edu \\ Mark Reese, ManTech International Corporation, mark.reese2@mantech.com
}

\begin{abstract}
While information systems are bridges that connect functional areas of an organization, the underlying purpose and value in that connectivity is to improve decision making on all levels of the organization. It stands to reason that if organizational decision makers were afforded the use of more intelligent information systems, that decision making would improve, and thrust the organization into a position of competitive advantage. Based on analyses conducted in 2004, in which investigators blended similar disciplines in order to build a conceptual framework and guidelines to ensure research quality and rigor, this proposition for further research purports an integration of these same disciplines to be used in the design of organizational information systems. The premise is that a more intelligent design could offer a higher level of pragmatic effectiveness in the system that is implemented [18]. Intelligence in design, here is twofold; one being a more naturalistic(the way decisions are actually made as opposed to how they should be made) presentation of information offered; and second, that the presentation is in a higher level of context (less information but more closely related to the actual decision at hand) [19, 14].
\end{abstract}

Keywords: Information Systems (IS), Decision Making, Design Science and Decision Science

\section{INTRODUCTION}

Half a century's worth of textbooks related to computer-based information systems have ingrained in us the mantra that, for these systems to be of worth to an organization, they, "must be able to provide the right information to the right people in the organization within the right time frame" [30]. Today's mantra is extended just a bit to include the reason for this accurate and timely information to "fuel smart decision making" [30]. Automation, control, and integration have become standard components to information systems within all organizations. As such, the attainment of these criteria alone does not better an organization's ability to make good decisions.
Up to now, information systems have been built on just that - the information. In an organization, the information can be compiled, massaged and summarized; however, it is, still, a somewhat stale representation of information offered to decision makers, who are then forced to alter their natural decision making process to follow the path created by the information. Instead of augmenting the cognitive capacity of the decision maker, it can limit the creative potential and/or the degree to which complex problems can be analyzed, resulting in a limited array of potential outcomes.

Taking this idea one step farther, if the value of information systems today is defined by their ability to integrate information entity-wide and present it, real-time to decision makers (i.e., ERP Systems), and if that has become the standard of corporate information systems today, how does that make one organization more competitive than another [10]? It seems reasonable that the new era of gaining competitive edge through information systems would have to lie in offering a more intelligent design and presentation of information to decision makers.

The synthesis and presentation of the information must encapsulate some degree of minimal intelligence so as to promote, not only more accurate decision making, but faster decision making with at least the same level of accuracy or higher. The potential value added here is that the ability to make faster, more accurate decisions than those of respective competitors affords a competitive advantage to the organization. While this is true, we subscribe to theorists who, despite all the accolades received by advancements in computer intelligence and capabilities, purport that the creativity of the human mind is still the arena where pure evolution and advancements are made [29]. The basic premise is quite simply that "since data is usually available to everyone, it is the creativity with which an individual can look at the data that makes the big difference. Creativity is the competitive tool that matters most" [12].

In following this line of thought, it seems reasonable to assume that if we understand decision making in a 
model that more closely reflects the reality of human decision making than the classical decision theories, we could better augment the organizational decision makers' capabilities.

Utilizing this understanding within the context of design science could then offer a more intelligent presentation of information that relates to the decision maker's problem space - used here to mean the context in which a decision must be made [23]. This could permit the professional a 'jump start' in the decision-making process, affording him/her an allowance to employ more creative thinking during decision making, or the attainment of decisions in more complex problem situations within the same timeframe.

We propose the exploration of design and presentation of the data of corporate information systems, through the integration of decision science and design science that could eliminate the rudimentary and preliminary steps in the decisionmaking process, primarily the selection and filtering of data into information as related to the context of the problem space, in order to afford decision makers time - that they would not otherwise have - to incorporate human creativity into their decision making.

\section{FOUNDATIONS OF PARADIGMS}

\section{Information Systems}

Starting with the role of an enormous adding machine for the U.S. Census Bureau in 1951, the evolution of computers and their use has evolved more rapidly than even Murphy's prediction, then thought of as an exaggeration, could imagine [31, 32]. Today, computer information systems have evolved to infiltrating almost every area of a business organization and work in concert to coordinate all the resources, information, and activities needed to complete business processes, and to assist the decisions that need to be made by employees, throughout the execution and completion of those processes. As previously stated, this mostly involves providing the right information to the right people in the right time frame so that they can make good decisions.

The potential value added by a more extensive use of information systems have been demonstrated in a number of fields, such as with military command and control and in medical diagnoses and patient care [16, 33]. In general terms, these systems contribute more to the decision maker than simple information systems because they perform a preliminary filtering of options and offer the decision maker a more finite solution set on which to begin the decision making process.

Using this value-added approach may allow businesses who are serious about using information systems to gain a competitive advantage through improved decision making and creativity, via these kinds of innovate information systems. This is where understanding the circumstances in situations in which decisions are actually made (what we call a problem space) as opposed to the way they (perhaps) should be made becomes key and moves us into the consideration of decision sciences in the information system design.

\section{Decision Science}

Some authorities trace the beginnings of decision theory to more than 300 years ago and begin a hierarchy of paradigms starting with "the distinction between normative and descriptive decision theories [as being], in principle, very simple. A normative decision theory is a theory about how decisions should be made, and a descriptive theory is a theory about how decisions are actually made" [15]. Not necessarily in contrast with this assumption, others mark the work of economists and statisticians over the last 50 years as the founding of decision theory, delineated with the prescriptive paradigm that "exploits some of the logical consequences of normative theories and empirical findings of descriptive studies" with the additional component of transitivity, where people make choices in a transitive fashion [4]. The emphasis in prescriptive theory is in "prescribing" what decision makers should do, and not at all understanding what it is that they, in fact, execute or how they diagnose in order to reach the point of execution [4]. These more traditional branches of decision science make up what is commonly termed as the "the first generation work of decision science" $[2,3]$.

The majority of traditional decision support systems and expert systems employed some form of prescriptive theory founded in economics and probability, where models were applied to decision making in order to "prescribe" what it is that decision makers should do. The focus of these early systems was in making a choice within the utilitarian paradigm to strive for "maximum utility" [17]. Gaming, choice and fuzzy group theories, to name a few, assume super rationality; and like their Bayesian predecessors assume to "perform better than a human 
expert" while ignoring influential, non-quantifiable variables in the domain $[7,17,28]$.

Viewed as a realistic shortcoming, the lack of knowledge as to the process behind decision making, and the ways in which a deviation in the process can alter the outcome, made way for the influences of psychology into the decision theory paradigms, that ultimately gave birth to Behavioral Sciences. Specialties such as Organizational Behavior have stemmed from Behavioral Sciences, and have become the generally-accepted schools which "attempt to more realistically describe how decisions are actually made, notably by professional decision makers such as managers" [3]. This second generation of decision science, also called Naturalistic Decision Making, abandons the restrictions of "choice" and "gambling" and, instead, "views decision making as a form of problem solving" $[3,8]$.

This is the fundamental tie between decision science and information systems; or more specifically, between Naturalistic Decision Making theory and its employment in the design of the information systems used in decision making.

Naturalistic Decision Making is a means of studying how people actually make decisions and perform cognitively complex functions in demanding situations. These include situations that are marked by time pressure, uncertainty and changing conditions, organizational constraints and varying amounts of experience [19].

Simply stated, these are real-world situations, (albeit defined as artificial by Simon et al.), requiring decisions, via human cognition, that will change the current situation, either in a positive or negative way. In any case, it will alter the problem space to progress to the next stage of decision making (and this could mean progressing forward or branching backward depending on the quality of the decision) or it will eliminate the problem at hand all together.

"Naturalistic Decision Making, a paradigm that examines the interplay of context and cognition together", provides a framework in which the design of information systems can offer a higher level of context in the information presented to support the decision maker, affording more room for the human cognition component in the process [19].

Within this concept, context is related to the familiarity the decision maker has with the situation or problem space (i.e., the culture, goals and expectations), which is, in turn, correlated with the amount and type of information that is presented to the decision maker. The more familiar the decision maker is with the situation, the higher the context surrounding the situation and the less information necessitated in achieving a level of cognitive understanding. It is also fair to say that the higher the context, the less verbiage needed because the language chosen to present the information would contain more intrinsic meaning to the decision maker [14].

So an information system built upon this concept would assume a high-context decision situation, which changes the traditional approach to information system design. This is where design science offers a complementary tool set to decision science and information systems through alternative design processes. Tom Peters, a contemporary forerunner in design sciences states that "design may be our top competitive edge" and that it "should be at the heart of every business" [24].

\section{Design Science}

The design-science paradigm has its roots in engineering and the sciences of the artificial; it is fundamentally a problem-solving paradigm that seeks to create innovations that define the ideas, practices, technical capabilities, and products through which the analysis, design, implementation, and use of information systems can be effectively and efficiently accomplished $[26,18]$. The product or outcome of design science, commonly referred to as an artifact, can be physical or conceptual in nature, and should encompass the ideas and practices of real-world professionals or domain experts [18]. Design science requires shaping artifacts and events to create a more desirable future [21].

"The importance of design is well recognized in the IS literature" [18]. An important step in the process of design science research is demonstrating that existing IT artifacts are or are not adequate for a specified problem [21]. For example, intuitiveness of the interface to users and the overall system processes have been design considerations in information systems development for years; however, the focus of design, in terms of decision making, has been to provide the professional with any and all information that could pertain to the problem space. Sometimes, though, display or access to all information may blur or cloud decision making. Some items within a systemic design may naturally 
be able to be divided into sub-parts and parts that could be eventually brought together as a whole. However, there are some problems or aspects of design that are not easily decomposed, even if highly complex - these tend to be social, political, or emotional issues. The key to success is to understand which items may be designed in parts to reduce complexity, and which items must be retained intact to understand the full measure of implications. Our intention is to show that a more intelligent display of information may be achieved by first employing decision science to the problem space, and then design science to the presentation. This includes more than the design of the initial presentation; it also includes how the system is designed to 'drill into' or 'logically branch' to consecutive presentations that are determined through the naturalistic study of the decision in conjunction with domain expertise. This brings us to the integration of theories.

\section{THE LOGICAL BLEND OF DECISION AND DESIGN SCIENCE WITH INFORMATION SYSTEMS DESIGN}

As previously stated, an exploration in the blending of these disciplines was conducted by a group of researchers in 2004, with the goal of building a "high-quality design-science research" that included "a concise, conceptual framework and clear guidelines for understanding, executing, and evaluating the research" [18]. Instead of continuing with the line of investigation started by these researchers to ensure research quality and rigor, we aim to explore the confluence of these sciences as a means to achieving a more intelligent design of information as it relates to the problem space. We purport that the attaining of a higher level of pragmatic, information-system effectiveness could afford professionals an opportunity for more than simply reaching an acceptable decision.

A brief exploration through military information systems will show a field which continues to extensively research the use of naturalistic decision sciences in information systems to augment the abilities of decision makers.

One such example is the study of the effects of organizational culture on the military decision making process. In [13], the authors investigate two military decision making models that involve information operations in order to determine the factors that are involved in choosing one model over another.
Another example is the digital "Battle Command Knowledge System" that is being explored to "meet global threats that are dynamic, innovative and adaptive" in military decision making [33] in a network centric warfare environment. The idea is that in the heat of a military threat, a battle captain is continually seeking information that relates to an ever-changing, unpredictable problem space. The result is an information overload that diminishes the leader's ability to reach sustainable, optimal decisions. To counter this, the use of a knowledge base is incorporated. The decision process of seasoned commanders and subject matter experts are used as input to a knowledge base. This knowledge base is used as the first of a series of decisions, which can be automated (automatable decisions) via coding of preset algorithms from the physical domain into the information domain. The second set of decisions, simple decisions, also relies upon codification of the knowledge base, along with some level of cognition. Given that the information system can assist with these first two decision types allows for more human intervention and cognition for the third decisionmaking area, complex decisions [1]. In essence, what occurs is that the information system performs the preliminary filtering process, that all decision makers first undergo, which then affords the commander the time and mental capacity to better critically evaluate the options for execution of the most complex steps or decisions.

This is a critical point of understanding. It is this second generation of decision theories, or naturalistic decision making, that encompass more real-world variables than its predecessors, to include such influences as culture, ethical underpinnings, group pressures, and even the intellectual limitations and current emotional states of decision makers. For example, in following image theory, a naturalistic decision process, whether or not a decision is carried out is dependent upon the personal goals, values, and strategies of the decision maker [22]. Some may even continue to pursue an irrational course of action because of previous investment, a phenomenon known as escalation [11]. This is the messy reality of decision making that, to-date, cannot be attained in a pure model. This is where performing the initial, rudimentary filtering process assists in the decision making process - by putting the creative leap back into the mind of the decision maker [12]. At this point, the decision maker could make a transitional choice which would branch into a new series of options based on the current decision as an input [6]. The end result would be that either the decision maker would choose an existing solution, or they would create a new alternative solution after having 
learned the outcomes of executing existing solutions. Hence, bringing us to the value added in exploring this type of information system in corporate information systems, where higher-level, complex decisions are made.

Simon [25] argued that people do not always follow through to completion on perfectly logical decision making, due in part to bounded rationality, the fact that there are costs inherent to the decision making, measured in terms of time to produce a decision [20], and therefore, an alternative that is merely sufficient may suffice.

This departure from totally logical decision making has been recognized both in theory and practice, due to the 'wicked nature' - defined as complex and dynamic with a critical dependence upon human cognitive abilities (creativity) and human social abilities (teamwork) to produce effective solutions [18] - of real-world problems. Therefore, it may not be possible to determine, let alone explicitly describe the relevant means, ends or laws. Even when it is possible to do so, the sheer size and complexity of the solution space will often render the problem computationally infeasible. In such situations, the search is for satisfactory solutions, i.e., satisficing [26], without explicitly specifying all possible solutions. The design task involves the creation, utilization, and assessment of heuristic search strategies. That is, construction of an artifact that works well for the specified class of problems."

Our intention here is to employ design science after considering exploring the problem space through decision science, so that the artifact that is designed can incorporate intrinsic variables, such as values, strategic goals and culture, into the filtering heuristics.

\section{APPLIED TO INFORMATION SYSTEMS DESIGN - A PRACTICAL BUSINESS EXAMPLE}

This portion of the paper explores a business case, providing some examples of potential integration of decision and design science. We would invite others to add suggestions as this paper is reviewed and discussed.

We propose a three-step process to implement this strategy, consisting of requirements gathering, design and application.

\section{Requirements Gathering}

The better we can understand the decision maker (application of behavioral science), the better we can understand the knowledge base for filtering and categorizing information... "knowledge from various sources in a knowledge intensive domain can be modeled using principles of ethnography. This is achieved by bridging the symmetry of ignorance gap that exists between process owners and system developers" [9]. One way of achieving this is to model an information landscape, or infoscape. An infoscape is a way of framing or observing a specific environment, be it from a political, financial, legal, or other multiple perspectives [27]. For example, many companies have made conscious and consistent efforts to establish a corporate mission, vision and core values process that is periodically revisited. Within the context of these items are the framework from which the company, the customer, the workforce, and the community are viewed. Given that these items are usually strong statements that tend toward the ideal, it should be possible to program/codify these messages.

\section{Design}

In this step, Design Science is employed to present information in ways that integrate the core ERP data and presentations (normally related to cost-, schedule-, and performance-based metrics) or business intelligence data/projections with those aspects of requirements gathering that can be modeled. Access to probabilistic tools and techniques would be included, as well as heuristic tools. As part of the decision making process, solution sets could be segregated by complexity, with those choices that require no or little human intervention in one area and more complex decision sets in another area.

Separating the decision sets offers choices to the decision maker that allow for the simple problems to be solved more or less automatically, with those of greater importance highlighted as areas of concern. Within the more complex problem area, information is presented in frames that follow the decision maker's natural cognitive path to allow for more cognitive engagement that encourages flexibility, creativity and interpretation. With respect to this creativity, Bolman and Deal state, "We cannot count the number of times managers have told us that they handled some problem the 'only way' it could be done. Such statements betray a failure of both imagination and courage" [5]. 


\section{Application}

The system design would permit the progression of frames or perspectives to allow for display of pertinent information, as well as the potential for "what if" scenarios that could be either probabilistic or non-probabilistic in nature. Given that the system could provide "solutions" for the simple problems, the cognitive domain could be engaged in solving problems with greater innovation.

Some of the decision making problems that could be addressed include:

- A manager must choose the focus of product development (internal research and development dollars). Are assets better spent continuing to enhance current products or is it better to look in a new technology area? [This could be a rather complex decision; however, access to and incorporation of corporate vision, direction and the long-term goals, along with market projections, may assist in making the decision or allow for innovation and integration of product lines.]

- A manager must select employees for hire/promotion. When making a selection, do you take the less experienced, motivated person, or the more experienced person that can do the job, but may not be motivated? [Again, this could be a complex business decision, especially if building a large workforce, with multiple factors to consider.]

- Where are training dollars best utilized? Given that a manager has an Information Assurance team, a help desk team and a network administration team, with only enough training funds to send people from one team, which is the best choice? [This could be based on total revenue, profit margin, etc.; however, it could also be based upon long term vision for the company to expand in one area or another, as well as most critical needs to satisfy a specific customer.]

- A manager has only two experienced Software developers and 3 tasks to accomplish. Does he/she commit each of them full time to one task and let the least risky task "find its own way," or does he/she set up some sort of rotational schedule to allow all three tasks to be overseen by the two experts, potentially resulting in three tasks completed to a slightly lower standard? [An enhanced design could involve such things as real-time modeling that allows for a simulation of performance results across a number of choices, with some weighted factoring of long term corporate goals and objectives. This preliminary solution development may allow for a better result for a less than optimal situation.]

\section{FUTURE RESEARCH}

The intent of this paper was to bring these issues to the collective information systems design and user community and to determine if there is a compelling reason for further research in the area of integrating naturalistic decision making. Can we expect to improve the capability of applications to share in the cognitive workload (outside of the artificial intelligence framework, which is mainly probabilistic in nature)? If so, what areas should be considered first?

\section{CONCLUSION}

In this paper, we propose the improvement of corporate decision making through the use of more intelligent information systems. These information systems differ from the 'traditional' information systems in that they integrate decision science and design science in an attempt to eliminate the rudimentary and preliminary steps in the decision making process. The intention of this treatise, presented to a cohort of peers, is to solicit professional and scholarly opinions from professionals and academicians as to the value of further research via a practical application.

\section{REFERENCES}

1. Alberts, D., Garstka, J., Hayes, R., \& Signori, D. (2004). Understanding information age warfare ( $3^{\text {rd }}$ Ed.). Washington, D.C.: Department of Defense Command and Control Research Program.

2. Beach, L.R. (1998). Image Theory: Theoretical and Empirical Foundations. New York, NY: Routledge.

3. Beach, L.R. \& Connolly, T. (2005). The Psychology of Decision Making: People in Organizations (Foundations for Organizational Science) ( $2^{\text {nd }} E d$.). Thousand Oaks, CA: Sage Publications.

4. Bell, D.E., Raiffa, H., \& Tversky, A. (1988). Decision Making: Descriptive, Normative and 
Prescriptive Interactions. Cambridge, UK: Cambridge University Press.

5. Bolman, L.G. \& Deal, T.E. (2008). Reframing Organizations Artistry, Choice and Leadership $\left(4^{\text {th }} E d\right.$.). San Francisco, CA: Jossey-Bass.

6. Debono, E. (1990). Lateral Thinking for Management: A Handbook of Creativity $\left(1^{\text {st }}\right.$ $E d$.). New York, NY: McGraw Hill.

7. Description. Fuzzy Optimization and Decision Making. Retrieved March 24, 2009, from http://www.springer.com/math/journal/10700

8. Falzer, P.R. (2004). Cognitive schema and naturalistic decision making in evidence-based practices. Journal of Biomedical Informatics, $37(2), 86-98$.

9. Fernandes, K.J. (2009). Interactive situation modelling in knowledge-intensive domains. International Journal of Business Information Systems, 4(1), 25-26.

10. Gao, J., Zhang, L., \& Jiang, W. (2007). Procuring requirements for ERP software based on semantic similarity. Proceedings of the $1^{\text {st }}$ IEEE International Conference on Semantic Computing (ICSC), Irvine CA USA.

11. Garland, H. (1990). Throwing good money after bad: The effect of sunk costs on the decision to escalate commitment to an ongoing project. Journal of Applied Psychology, 75, 728-731.

12. Gedeon, T.D. \& Wynter, M.C.P. (1994). Explaining creatively. In Dingli, S. (Ed.), Creative Thinking: A Multifaceted Approach (191-200). Malta: Malta University Press.

13. George, L., Grant, A. J., Jabro, A. D., \& Skovira, R. J. (2008). Synchronizing knowledge in military decision making: A research approach for exploring the effects of organizational culture. Issues in Information Systems, IX(2), 429-438.

14. Hall, E.T. (1989). Beyond Culture. New York, NY: Anchor Books, Doubleday.

15. Hansson, S.O. (1994, 2005). Decision theory a brief introduction. Retrieved March 24, 2009, from http://www.infra.kth.se/ soh/decisiontheory.p df

16. Haux R. (2006). Health information systemspast, present and future. International Journal of Medical Informatics 75(3-4), 268-281.

17. Henrion, M., Breese, J.S., \& Horvitz, E.J. (1991, Winter). Decision analysis and expert systems. AI Magazine, 12(4), 64-91.

18. Hevner, A., March, S., Park, J., \& Ram, S. (2004). Design science in information systems research. MIS Quarterly 28(1), 75-105.

19. Klein Associates. Applied Research Associates. Retrieved June 29, 2009, from www.decisionmaking.com or www.ara.com

20. Lu, H.C., Chen, M., \& Chang, J.J. (2005) Are more alternatives better for decision-makers? A note on the role of decision cost. Theory and Decision, 58: 283-304.

21. March, S. T. \& Storey V. C. (2008). Design science in the information systems discipline: An introduction to the special issue on design science research. MIS Quarterly 32(4), 725-730.

22. Morrell, K. (2004). Decision making and business ethics: the implications of using image theory in preference to rational choice. Journal of Business Ethics 50(3), 239-252.

23. Newell, A. \& Simon, H. (1972). Human Problem Solving. Englewoods Hills, NJ: Prentice-Hall.

24. Peters, T. Tom Peters Professional Web Site. Retrieved March 24, 2009, from http://www.tompeters.com/

25. Simon, H.A. (1955). A behavioral model of rational choice, Quarterly Journal of Economics, 69, 99-118.

26. Simon, H.A. (1996). The Sciences of the Artificial $\left(3^{\text {rd }} E d\right.$.). Cambridge, MA: MIT Press.

27. Skovira, R. J. (2004). Using informational landscape as a model to understand information use and design within organizations. Issues in Information Systems $1,308-314$ 
Blending Decision and Design Science with Information Systems Design as a Means to Optimizing Business Decisions

28. Skyrms, B., (1990). Dynamic models of deliberation and the theory of games. Proceedings of the $3^{\text {rd }}$ conference on Theoretical Aspects of Reasoning about Knowledge, Pacific Grove CA USA.

29. Soh (2008). Getting a real return on your ERP investment. Retrieved April 10, 2009 from http://marketing-interactive.com/news/6686

30. Stair, R. \& Reynolds, G. (2007). Principles of Information Systems ( $8^{\text {th }} E d$.). Florence, KY: Course Technology.

31. Tuomi, I. (2002). The lives and death of Moore's law. First Monday, 7(11). Retrieved March 24, 2009 , from http://firstmonday.org/htbin/cgiwrap/bin/ojs/i ndex.php/fm/article/view/1000

32. U.S. Census Bureau. (2004). $53^{\text {rd }}$ Anniversary of the UNIVAC Computer. Retrieved March 24, 2009, from http://www.census.gov/PressRelease/www/releases/archives/facts for feat ures_special_editions/001784.html

33. White, A., Stawasz, J., O'Hare, S., Jenevein, R., Fussell, D. \& Darrell, W. (2005). Digital warrior: blending pedagogy and game theory. Proceedings of the Interservice/Industry Training, Simulation, and Education Conference (I/ITSEC), Orlando FL USA. 